Research Paper

\title{
In Surgical Colon Cancer Patients Extended-Duration Thromboprophylaxis (30 days) with the Highest Dose of Tinzaparin (4,500 IU s.c./q.d.) Normalizes the Postoperative VEGF Levels
}

\author{
Michail Mitsis ${ }^{1 \# 凶}$, Panagiotis Koliou ${ }^{\# 1}$, Christina Bali ${ }^{1}$, Evangelia Ntounousi ${ }^{2}$, Vasileios Tatsis ${ }^{1}$, Vasileios
} Nousias ${ }^{1}$, Georgios D Lianos ${ }^{1}$, Georgios Vartholomatos ${ }^{3}$ and Dimitrios Nastos ${ }^{1}$

1. Department of Surgery, University Hospital of Ioannina, Greece;

2. Department of Nephrology, University Hospital of Ioannina, Greece;

3. Unit of Molecular Biology of the Haematology Laboratory, University Hospital of Ioannina, Greece.

\#The first two authors (MM and PK) are co-first authors, as they have contributed equally to this study.

$\square$ Corresponding author: Michail Mitsis, MD, PhD, FEBS, Associate Professor of Surgery-Transplantation, Department of Surgery, University Hospital of Ioannina, S. Niarchos av., 45500 Ioannina, Greece. Tel: +32 6510 99696; mob.:+32 6948047917; Fax.:+32 6510 99890; E-mail: mmitsis@cc.uoi.gr

(c) Ivyspring International Publisher. This is an open access article distributed under the terms of the Creative Commons Attribution (CC BY-NC) license (https:// creativecommons.org/licenses/by-nc/4.0/). See http://ivyspring.com/terms for full terms and conditions.

Received: 2017.03.16; Accepted: 2017.06.30; Published: 2017.08.25

\begin{abstract}
Background/Purpose: In colon cancer (CC) patients preoperative (pre-op) levels of VEGF-A 165 (VEGF) is a strong predictor for disease recurrence. Elevated postoperative (post-op) VEGF levels could have undesirable effects by enhancing tumor growth and metastasis formation. It has been suggested that thromboprophylaxis with a Low Molecular Weight Heparin (LMWH) in surgical cancer patients, further to thromboembolic protection, may exert some anti-neoplastic properties, as well. The aim of our study was to assess the potential impact of the LMWH Tinzaparin (Innohep ${ }^{\circledR}$ - Leo Pharma, Copenhagen, Denmark), given at different doses and for different perioperative (peri-op) periods, upon the post-op variability of serum VEGF levels in surgical CC patients.

Methods: A total of 54 consecutive $C C$ patients who underwent a curative resection were randomized in four groups according to their peri-op thromboprophylaxis scheme, which was based on administrating Tinzaparin in different doses and at different periods, as follows: group I: 3,500 IU for 10 days, group II: 3,500 IU for 30 days, group III: 4,500 IU for 10 days and group IV: 4,500 IU for 30 days. Serum VEGF concentrations were evaluated on the pre-op day (Day 0 ) and on the $10^{\text {th }}$ and $30^{\text {th }}$ post-op days (Day 10 and Day 30, respectively). For statistical analyses the mixed design ANOVA was used. $\mathrm{P}<$ 0.05 was considered significant.

Results: On Day 0, VEGF didn't differ between groups I, II, III and IV ( $p>0.05$, for every comparison). On Day 10, VEGF was increased in all groups. Between Day 10 and Day 30, VEGF remained stable in groups I $(p=0.031)$ and II $(p=1.000)$ and increased significantly in group III $(p=0.005)$. On the contrary, VEGF decreased significantly in group IV $(p<0.001)$. The most remarkable finding was observed when we compared VEGF between Day 0 and Day 30: while in groups I, II and III, VEGF remained significantly higher compared to Day 0 ( $p<0.001, p=0.041$ and $p<0.001$, respectively), on the contrary, in group IV (extended-duration with the highest dose of 4,500 IU of tinzaparin) it was comparable to Day 0 $(p=1.000)$.

Conclusions: In surgical CC patients only the recommended thromboprophylaxis scheme with the highest prophylactic dose of Tinzaparin (4,500 IU) for extended-duration (30 days) normalizes VEGF levels at the end of the first post-op month by reducing them to the pre-op levels.
\end{abstract}

Key words: colon cancer; tinzaparin; thromboprophylaxis; VEGF; angiogenesis. 


\section{Introduction}

Colon cancer (CC) is the commonest form of colorectal cancer representing about $72 \%$ of all cases. Surgery with a curative intention is the standard of care for these patients. Nevertheless, even after an apparently curative tumor resection a significant proportion of patients develop local or distant recurrence of the disease. Thus the 5-year relative survival rate for patients staged IIa is $87 \%$ falling to only $53 \%$ for patients staged IIIc [1]. We know today that disseminated cancer cells can persist for long periods in a dormant state (either as minimal residual disease or micrometastases) below the threshold of diagnostic detection, surgical intervention and evade therapies [2-4]. Angiogenic dormancy significantly contributes to this behavior by keeping a net balance between the cell proliferation rate and an equivalent rate of cell death/apoptosis [5]. When the angiogenic factors overcome their antagonists then the so called "angiogenic switch" turns on and transforms the almost avascular micrometastases to a well vascularized and growing tumor mass $[6,7]$.

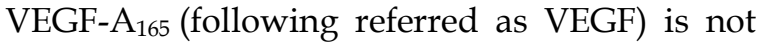
only the most potent, direct acting angiogenic protein known, but it also plays a pivotal role in many normal physiological functions including wound healing process [8]. Several studies in CC patients have suggested that VEGF is a strong and sensitive predictive marker for disease recurrence and overall survival [9-12]. Although, a few studies examined the postoperative (post-op) variability of VEGF concentrations, it seems that they are persistently elevated for at least up to the first post-op month [13]. The clinical importance of this elevation is uncertain, but it could be a potentially harmful event stimulating residual tumor cells growth. For non-metastatic CC patients the use of specialized anti-VEGF agents in the adjuvant setting didn't demonstrate improved overall survival or relapse-free survival, but we must keep in mind that in all these series surgery occurred 4-8 weeks before the patients' randomization [14]. An equal delay in every adjuvant treatment is always necessary due to the antiproliferative properties of the chemotherapeutic agents which affect, amongst others, wound and anastomoses healing. Since, it is known that small tumor foci and micrometastases tend to grow fast and additionally that the post-op period is a dangerous time for cancer patients who harbor residual cancer, then this "non-chemotherapy" period could represent an "Achilles heel" in the modern multimodal treatment of cancer [15-17].

In recent years, extended-duration (4 weeks) thromboprophylaxis with the highest prophylactic dose of Low Molecular Weight Heparin (LMWH) is recommended for most of the patients who undergo abdominal or pelvic surgery for cancer, because this scheme has been shown to offer the highest level of protection against the development of thromboembolic events during the post-op period $[18,19]$. Several studies have suggested that these compounds except for their antithrombotic actions may have additional significant antineoplastic properties, as well $[20,21]$. Tinzaparin (Innohep ${ }^{\circledR}$ Leo Pharma, Copenhagen, Denmark) is a widely used LMWH and apart from the ability to inhibit thrombin and factor $\mathrm{Xa}$ it particularly shares most of the above mentioned anti-neoplastic properties [22]. The purpose of our study was to evaluate the potential impact of this compound when administered in different doses and/or different periods upon the post-op variability of the VEGF concentrations in CC patients who underwent a curative surgical resection.

\section{Patients and Methods}

\section{Study design and participants}

During a 36 month-period all consecutive patients with endoscopic biopsy-proven CC planning colectomy with curative intent at the Surgical Department of Ioannina University Hospital, were considered for their eligibility to enter the study. Patients with conditions which possibly could have been implicated in VEGF production, such as a history of: any type of malignancy other than CC, diabetes mellitus, venous thromboembolic events, coronary or peripheral vascular disease, systematic reception of anticoagulant and/or antiplatelet agents and severe renal insufficiency, were exclude from the study. Moreover, it was predetermined that patients who would be found during the progress of the study to be homogenous for Factor $\mathrm{V}$ Leiden or Prothrombin G20210A would be excluded, because there is strong evidence that homozygous individuals for these two mutations generate more thrombin compared to non-carriers and increased thrombin concentrations lead to increased activation of platelets, and consequently, to increased VEGF production [23,24]. Furthermore, we excluded patients with the presence of distant metastasis found intraoperatively (no R0 resection), with a final histopathologic report not confirmative for cancer and finally, patients who developed post-op complications or failed to complete data collection. In an attempt to achieve a number of fifteen patients per group the allocation was completed in consecutive 
phases. Sequential patients entered the first group until its completion and then the following patients entered the next group, and so on. After providing written informed consent, patients were assigned to receive perioperative thromboprophylaxis with Tinzaparin (s.c./q.d.) with the first dose given two to four hours pre-op. The patients were allocated in the four groups of the study according to the thromboprophylaxis scheme as follows: group I: 3,500 I.U. for 10 days, group II: 3,500 I.U. for 30 days, group III: 4,500 I.U. for 10 days and group IV: 4,500 I.U. for 30 days.

All the clinical, laboratory and histological data of every patient were collected and recorded on a data base sheet. All patients were staged for their disease according to the American Joint Committee on Cancer TNM staging system. The procedures followed were in accordance with the ethical standards of the Declaration of Helsinki and approved by the Scientific Committee of the University Hospital of Ioannina, Greece.

\section{Blood sampling and assays}

Except for the conventional laboratory investigations, full blood samples were taken from each patient on Day 0, collected in EDTA tubes and stored for the evaluation of heritable thrombophilia. Factor V Leiden and Prothrombin G20210A mutations were tested using multiplex allele-specific polymerase chain reaction amplification. For the determination of serum VEGF concentrations full blood samples were collected in plain tubes at three different time-points: Day 0, Day 10 and Day 30. Then they were centrifuged $\left(20 \mathrm{~min}\right.$, at $3,000 \mathrm{r} / \mathrm{min}$, at $\left.4{ }^{\circ} \mathrm{C}\right)$ without the use of centrifuge break to avoid any mixing of the clotted material. Subsequently, serum was separated by aspiration and stored in polyethylene tubes at -80 ${ }^{\circ} \mathrm{C}$ before analysis. Serum VEGF165 levels were determined in duplicate via commercially available enzyme-linked immunosorbent assay (ELISA - R\&D Systems Inc., Minneapolis, MN) according to instructions. The results were read using an automated microplate reader (LabSystems Multiskan RC/MS/EX Microplate Reader, Artisan Technology Group, Champaign, IL). Concentrations are reported as $\mathrm{pg} / \mathrm{mL}$. Throughout the study period blood samples were regularly taken from every patient for monitoring of any possible adverse effects related to the administration of tinzaparin (fall of Hemoglobin/ Hematocrit, Thrombocytopenia, hyperkalemia, increase of Liver transaminases).

\section{Data analysis}

The Statistical Package for Social Sciences (SPSS) v21 (SPSS Inc, Chicago, IL, USA) for Windows was utilized for all statistical analyses. Frequencies and percentages were determined for categorical variables whereas clinical and demographic data are expressed as the mean and standard deviation for continuous variables. For the comparison of serum VEGF at each time-point of blood sampling (Day 0, Day 10, Day 30) for any intra and inter-group differences repeated measures of mixed design ANOVA were used. Post-hoc analyses were performed using the Tukey's range test. A $P$-value less than 0.05 was considered statistically significant. In order to investigate the impact of different doses or different periods of Tinzaparin's administration upon VEGF concentrations we formed four consolidated groups (CGs) by combining patients from different groups. The first consisted of patients from groups I and II who received the conventional dose of Tinzaparin (CG-CD: 3,500 IU), the second were patients from groups III and IV who received the highest prophylactic dose (CG-HD: 4,500 IU), the third were patients from groups I and III who received short-duration prophylaxis (CG-SD: 10 days) and finally the fourth, were patients from groups II and IV who received extended-duration prophylaxis (CG-ED: 30 days).

\section{Results}

\section{Study population and demographics}

As it is shown in the Flow chart (Fig. A), a total of 112 consecutive CC patients were considered for eligibility. 36 out of 122 were not found to fulfil the inclusion criteria and they were excluded. 76 patients were found to be eligible for participation. During the progress of the study 17 more patients were additionally excluded: 4 patients (one from each group) developed post-op complication (a wound infection in group I, a post-op peritonitis in group II, a pancreatitis in group III and a pneumonia in group IV), 3 patients were found to be carriers of hereditary thrombophilia, 3 patients failed to data collection, 1 patient was found intraoperatively with a liver metastasis and finally in 6 patients the histopathologic report did not confirm the initial diagnosis of cancer shown in the Flow Chart). Thus, the number of the 59 allocated patients in each group was as follows: group I: 16, group II: 15, group III: 13 and group IV: 15 .

During the data analysis five patients were found to express postoperatively extremely high levels of VEGF, which were not in accordance with the rest patients. A deeper analysis revealed that these patients, who initially had all been included in group I, manifested the typical histological features of mucinous adenocarcinoma. This subtype is considered as unfavorable due to its poor response to 
commonly used chemotherapeutics and the potential for, and severity of, metastasis [18]. These five patients were excluded from group I and we comment on their findings as a separate cohort (referred bellow as MC pts: Mucinous Cancer patients). Thus, the number of patients who were finally allocated in the four groups of the study was 54: 11 in group I, 15 in group II, 13 in group III and 15 in group IV. The demographics (gender, mean age) and the staging of the patients are shown in Table 1 . There were no differences between the patients in terms of their demographics, as well as, in PLT count, aPTT and INR values (for the laboratory findings the data are not shown). More patients in groups III and IV compared to groups I and II had stage III disease (68\% vs. $46 \%$, respectively). From the regular laboratory and clinical investigation no adverse effects related to tinzaparin's administration were observed.

\section{Peri-operative variability of VEGF concentrations}

All serum VEGF concentrations are expressed as mean $( \pm S D)$ values in $\mathrm{pg} / \mathrm{mL}$ and along with the relative intra-group comparisons at the three time-points are shown in Table 2. Inter-group comparisons between the CGs are shown in Table 3 and between Day 10 and Day 30 for the main groups and the MC pts are shown in Table 4.

\section{CG-CD and CG-HD patients: Conventional vs. High dose of Tinzaparin}

No differences in serum VEGF levels were found between the two groups at any time-point $(p=0.330$, $p=0.230$ and $p=0.730$ for Day 0, Day 10 and Day 30, respectively). In both groups VEGF was significantly increased on Day 10 compared to Day 0 (both $\mathrm{p}<0.001)$. On Day 30, VEGF was similar to Day 10 $(p=1.000$ and $p=0.711$ for CG-LD and CG-HD, respectively), but it was constantly significantly higher compared to Day 0 ( $p<0.001$ and $p=0.008$ for CG-CD and CG-HD, respectively).

Table 1. Demographics and staging of the patients in the four main groups.

\begin{tabular}{lllll}
\hline & $\begin{array}{l}\text { Group I } \\
(\mathrm{n}=11)\end{array}$ & $\begin{array}{l}\text { Group II } \\
(\mathrm{n}=15)\end{array}$ & $\begin{array}{l}\text { Group III } \\
(\mathrm{n}=13)\end{array}$ & $\begin{array}{l}\text { Group IV } \\
(\mathrm{n}=15)\end{array}$ \\
\hline Gender n (\%) & & & & \\
Male & $6(55)$ & $7(47)$ & $6(46)$ & $7(47)$ \\
Female & $5(45)$ & $8(53)$ & $7(54)$ & $8(53)$ \\
$\begin{array}{l}\text { Mean Age (years } \\
\pm \text { SD) }\end{array}$ & $65( \pm 8)$ & $62( \pm 10)$ & $63( \pm 11)$ & $64( \pm 10)$ \\
Stages n (\%) & & & & \\
I & $1(9)$ & $1(7)$ & $1(8)$ & $0(0)$ \\
II & $6(55)$ & $6(40)$ & $3(23)$ & $5(33)$ \\
III & $4(36)$ & $8(53)$ & $9(69)$ & $10(67)$ \\
\hline
\end{tabular}

Table 2. Mean serum VEGF concentrations and intra-group comparisons between the three time-points.

\begin{tabular}{|c|c|c|c|c|c|c|}
\hline \multirow[t]{2}{*}{ Groups } & Day 0 & Day 10 & Day 30 & $\begin{array}{l}\text { Day 0/ } \\
\text { Day } 10\end{array}$ & $\begin{array}{l}\text { Day 10/ } \\
\text { Day } 30\end{array}$ & $\begin{array}{l}\text { Day 0/ } \\
\text { Day } 30\end{array}$ \\
\hline & \multicolumn{3}{|c|}{$\begin{array}{l}\text { mean serum VEGF concentrations in } \\
\mathrm{pg} / \mathrm{mL}( \pm S D)\end{array}$} & $\mathrm{P}$ & $\mathrm{P}$ & $\mathrm{P}$ \\
\hline I & $369( \pm 411)$ & $667( \pm 268)$ & $810( \pm 153)$ & 0.008 & 0.301 & 0.000 \\
\hline II & $471( \pm 228)$ & $703( \pm 331)$ & $685( \pm 276)$ & 0.017 & 1.000 & 0.041 \\
\hline III & $549( \pm 184)$ & $713( \pm 274)$ & $971( \pm 387)$ & 0.190 & 0.005 & 0.000 \\
\hline IV & $450( \pm 174)$ & $854( \pm 332)$ & $477( \pm 226)$ & 0.000 & 0.000 & 1.000 \\
\hline MC pts & $490( \pm 396)$ & $1,560( \pm 333)$ & $1,112( \pm 348)$ & 0.000 & 0.003 & 0.000 \\
\hline CG-CD & $428( \pm 315)$ & $688( \pm 300)$ & $738( \pm 237)$ & 0.000 & 1.000 & 0.000 \\
\hline CG-HD & $497( \pm 182)$ & $789( \pm 309)$ & $707( \pm 394)$ & 0.000 & 0.711 & 0.008 \\
\hline CG-SD & $467( \pm 315)$ & $692( \pm 266)$ & $898( \pm 308)$ & 0.003 & 0.006 & 0.000 \\
\hline CG-ED & $461( \pm 199)$ & $779( \pm 335)$ & $581( \pm 269)$ & 0.000 & 0.003 & 0.131 \\
\hline
\end{tabular}

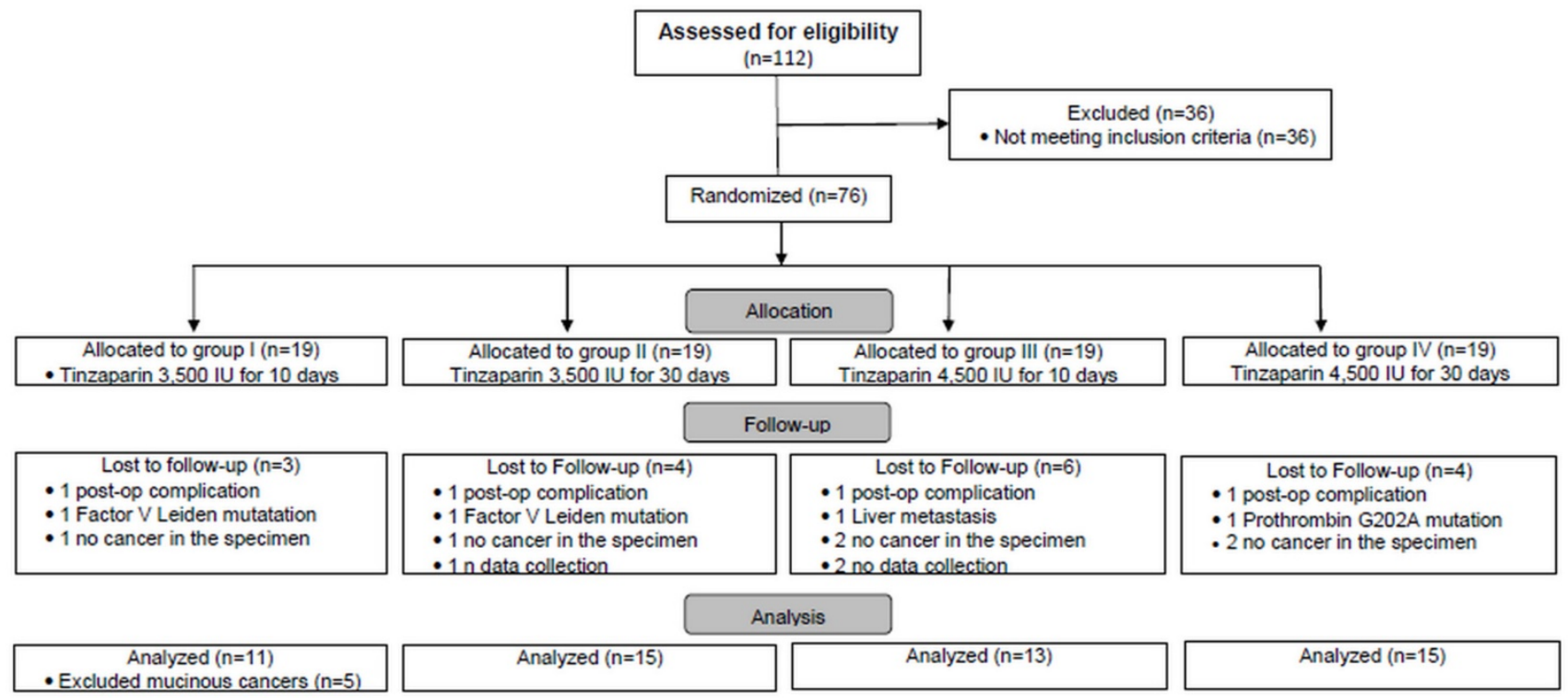

Fig. A. Flow chart. Eligibility, exclusion, randomization, allocation, follow-up and analysis of the study's population. 


\section{CG-SD and CG-ED patients: Short vs. Extended duration prophylaxis}

In both groups VEGF levels were significantly increased on Day 10 compared to Day $0(p=0.003$ and $\mathrm{p}<0.001$, for CG-SD and CG-ED, respectively). At both time-points VEGF was comparable between the two groups $(p=0.931$ and $p=0.306$ for Day 0 and Day 10, respectively). However, between Day 10 and Day 30 VEGF continued to increase in CG-SD $(p=0.006)$, while it was significantly decreased in CG-ED $(p=0.003)$. Thus, on Day 30, VEGF was significantly higher in the CG-SD compared to CG-ED $(p<0.001)$ and more interestingly only in the latter group it was comparable to Day $0(p=0.131)$.

Table 3. Inter-group comparisons of mean serum VEGF concentrations in Consolidated Groups.

\begin{tabular}{llrl}
\hline & \multicolumn{2}{l}{ Conventional Dose vs. Highest Dose } & \\
\cline { 2 - 3 } & \multicolumn{2}{l}{ CG-CD } & CG-HD \\
\cline { 2 - 4 } & mean VEGF concentrations $( \pm$ SD $)$ & 0.330 \\
Day 0 & $428( \pm 315)$ & $497( \pm 182)$ & 0.230 \\
Day 10 & $688( \pm 300)$ & $789( \pm 309)$ & 0.730 \\
Day 30 & $738( \pm 237)$ & $707( \pm 394)$ & \\
& Short-Duration vs. Extended-Duration & P \\
& CG-SD & CG-ED & \\
& mean VEGF concentrations $( \pm S D)$ & 0.931 \\
Day 0 & $467( \pm 315)$ & $461( \pm 199)$ & 0.306 \\
Day 10 & $692( \pm 266)$ & $779( \pm 335)$ & 0.000 \\
Day 30 & $898( \pm 308)$ & $581( \pm 269)$ & \\
\hline
\end{tabular}

Table 4. Inter-group comparisons of mean serum VEGF levels concentrations on Day 10 and Day 30.

\begin{tabular}{llllllll}
\hline Day 10 & & \multicolumn{7}{c}{ Day 30 } \\
\hline Group A & Group B & MD & P & Group A & Group B & MD & P \\
\hline I & II & -36.1 & 1,000 & I & II & 125 & 1,000 \\
& III & -46.2 & 1,000 & & III & -161 & 1,000 \\
& IV & -187.5 & 1,000 & & IV & 333 & 0,044 \\
& MC pts & -892.9 & 0,000 & & MC pts & -302 & 0,521 \\
II & I & 36.1 & 1,000 & II & I & -125 & 1,000 \\
& III & -10.1 & 1,000 & & III & -287 & 0,097 \\
& IV & -151.4 & 1,000 & & IV & 207 & 0,489 \\
& MC pts & -856.8 & 0,000 & & MC pts & -427 & 0,049 \\
III & I & 46.2 & 1,000 & III & I & 161 & 1,000 \\
& II & 10.1 & 1,000 & & II & 287 & 0,097 \\
& IV & -141.3 & 1,000 & & IV & 494 & 0,000 \\
& MC pts & -846.7 & 0,000 & & MC pts & -141 & 1,000 \\
IV & I & 187.5 & 1,000 & IV & I & -332 & 0,044 \\
& II & 151.4 & 1,000 & & II & -207 & 0,489 \\
& III & 141.3 & 1,000 & & III & -494 & 0,000 \\
& MC pts & -705.4 & 0,000 & & MC pts & -635 & 0,001 \\
MC pts & I & 892.9 & 0,000 & MC pts & I & 302 & 0,521 \\
& II & 856.8 & 0,000 & & II & 427 & 0,049 \\
& III & 846.7 & 0,000 & & III & 141 & 1,000 \\
& IV & 705.4 & 0,000 & & IV & 635 & 0,001 \\
\hline MD: & Mean Difference of mean values. & & &
\end{tabular}

Intra and inter-group comparisons within and between the four main groups

On Day 0, VEGF didn't differ between groups I, II, III and IV ( $p>0.05$, for every comparison). On Day
10 VEGF was increased in all groups. This increase was statistically significant in groups I, II and IV ( $p=0.008, p=0.017$ and $p<0.001$, respectively), except in group III $(p=0.190)$. Between Day 10 and Day 30, VEGF increased significantly in group III $(p=0.005)$ and decreased significantly in group IV $(p<0.001)$. In the other two groups it remained unchanged: $(p=0.301$ and $p=1.000$, for groups I and II, respectively). A remarkable finding was observed when we compared VEGF between Day 0 and Day 30 (see Figure 1). Whilst on Day 30 VEGF remained significantly higher compared to Day 0 in groups I, II and III ( $p<0.001, p=0.041$ and $p<0.001$, respectively), on the contrary, in group IV (extended-duration thromboprophylaxis with the highest dose of 4,500 IU of tinzaparin) VEGF was comparable to that on Day 0 $(\mathrm{p}=1.000)$.

\section{VEGF variability in MC pts}

In MC pts, pre-op serum VEGF was comparable to groups I, II, III and IV (all p>0.05). On Day 10, VEGF showed an excessive increase (more than 3 times) compared to Day $0 \quad(p<0.001)$ and was significantly higher compared to groups I, II, II and IV at the same time-point (all p<0.001). On Day 30, VEGF was shown to be significantly lower compared to Day $10(p=0.003)$ but it was still significantly higher compared to Day $0(\mathrm{p}<0.001)$.

\section{Discussion}

In the present study we assessed the perioperative variability of serum VEGF concentrations in CC patients and evaluated the impact of Tinzaparin upon this potent promoter of angiogenesis. VEGF has been widely studied in CC patients and has been suggested that it directly mediates CC growth and metastasis [25]. Thus, theoretically, a possible normalization of VEGF concentrations during the immediate post-op period could be a promising finding. The base-line levels of serum VEGF were comparable between the four main groups and they are in agreement with previous studies reporting increased levels of circulating VEGF in CC patients [25-27]. However, surgical trauma is associated with alterations of the angiogenic environment and thus the observed post-op VEGF increase probably reflects its hyper-production from several cells during the wound healing process following treatment-induced tissue injury. This observation could not be easily ignored because the post-op release of VEGF could have undesirable effects on residual tumor cells and may enhance tumor growth and metastasis formation. 


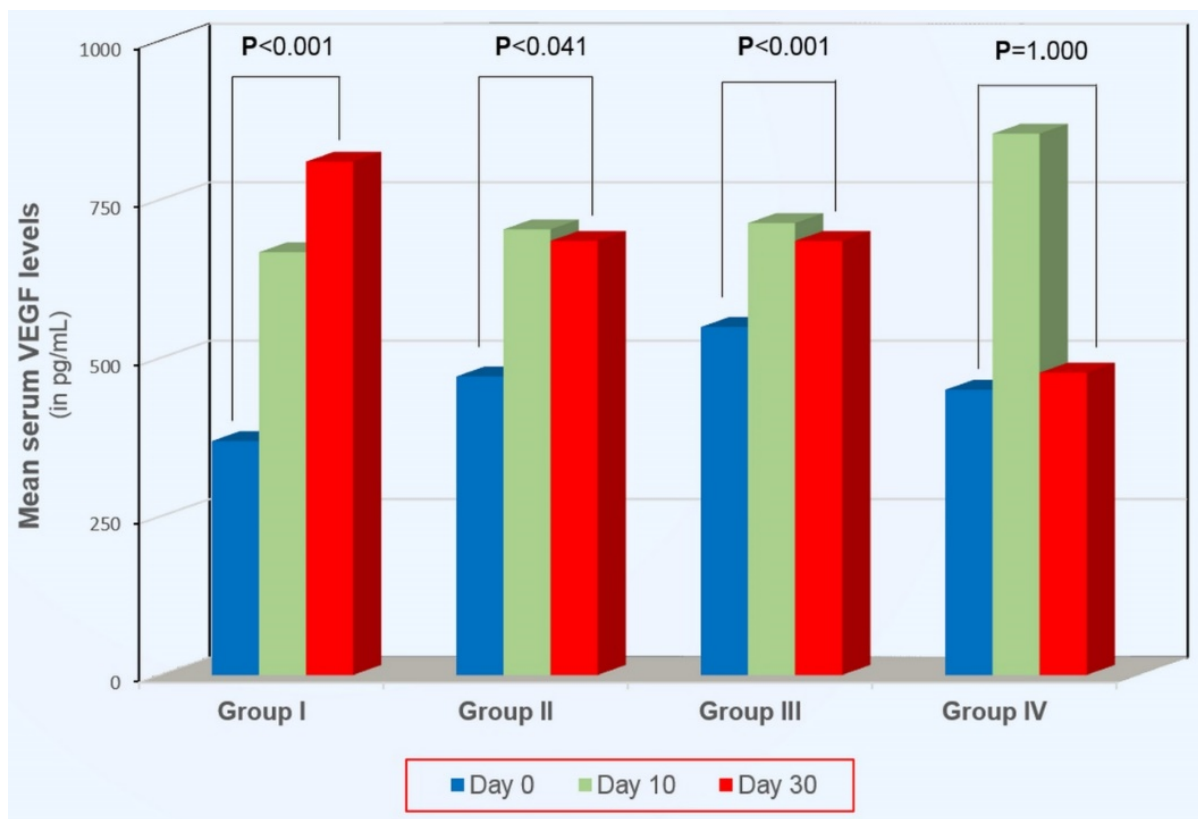

Figure 1. Intragroup comparisons of serum VEGF levels between Day 0 and Day 30.

A point of interest is that on Day 10, VEGF was significantly increased in all main groups except for group III. The patients of the latter group received the highest prophylactic dose of Tinzaparin and thus it could be supposed that this highest dose showed a possible protective effect. However the same dose was given to patients of group IV as well, but the same effect was not apparent. Additionally, during this first post-op period (Day 0 to Day 10) the absence of any effect of the highest dose of Tinzaparin upon VEGF levels was observed when we compared patients of CG-HD to patients of CG-CD. A first explanation could be that even the highest prophylactic dose was too weak to control VEGF elevation at least to all patients. Nevertheless, it is known that CC patients with advanced stages of their disease show higher VEGF concentrations and in our study's population the impact of the highest dose could have been undermined by the larger proportion of staged III patients in CG-HD compared to CG-CD. More homogenous studies in terms of patients' staging could clarify this issue.

The discontinuation of Tinzaparin from Day 10 to Day 30 resulted in elevation of VEGF levels. This was more obvious in patients who had received the highest dose (group III) than in patients with the conventional dose (group I). Although a rebound phenomenon cannot be ruled out, again the disproportion between the two groups in terms of disease staging does not permit any definite conclusions.

On the contrary, the continuation of Tinzaparin's administration during the same period (Day 10 to Day
30) was shown to decrease VEGF levels. This effect led to a very interesting result particularly in patients who received the highest dose of Tinzaparin (group IV). In these patients a restoration of Day 30 VEGF levels down to the pre-op levels was achieved. This finding suggests that the current recommended thromboprophylaxis scheme (administration of the highest prophylactic dose for extended-duration) offers not only the best protection against the development of thromboembolic events, but it also adds the very promising benefit of normalizing VEGF concentrations at the end of the first post-op month by bringing them down to the pre-op levels. As far as we know, this finding is unique in the accessible international literature.

This above mentioned normalizing effect of Tinzaparin upon the perioperative VEGF variability is not easily explained, but mechanisms related to its pleotropic actions could be possibly proposed. The most of the circulating serum VEGF is clearly due to its release from platelets during the clotting process and nowadays these cells are recognized as the major source of VEGF as the platelet pool comprises more than $80 \%$ of total circulating VEGF in cancer patients and in healthy individuals, as well $[28,29]$. CC patients, as almost all patients with solid malignancies, manifest a persistent activation of their haemostatic mechanisms [30]. It has been shown that Tinzaparin down-regulates the tumor-associated hypercoagulability through various means and thus it may lead to amelioration of VEGF production [22]. For example, it exerts its main antithrombotic action through the inhibition of thrombin and factor Xa. 
Thrombin's inhibition decreases both the motility of endothelial cells and the activation of platelets, and consequently, VEGF production is limited [31]. It is known that cancer progression is accompanied by platelet activation and some recent findings have proposed Tinzaparin to be the most potent inhibitor of thrombin generation compared to other LMWHs $[32,33]$. Additionally, Tinzaparin suppresses Tissue Factor (TF) expression and induces maximum Tissue Factor Pathway Inhibitor (TFPI) release which contributes to a further inhibition of TF activity. TF is not only involved in the initiation of the extrinsic pathway of the coagulation cascade, but it also promotes tumor angiogenesis and metastasis via VEGF production, while, on the contrary, TFPI exerts a possible protective effect $[34,35]$. Moreover, other actions of LMWHs in general, such as, the inhibition of cell-cell-interactions (through blocking of P- and L-selectins), the inhibition of extracellular matrix protease heparanase and a potential binding affinity to VEGF may all contribute to their anti-neoplastic effects $[36,37]$. Finally, we think that the finding of the excessive VEGF increase during the post-op period in the patients with mucinous adenocarcinomas should not be underestimated. Even though the small number of relative cases in our study's population does not permit any definite conclusions, it seems that VEGF overproduction is consistent with the accepted perception that this histologic subtype of CC possibly represents a distinct biological entity [38].

\section{Conclusions and limitations}

Although surgical intervention remains the standard of care for CC patients, at the same time it may contribute to an increase in the most potent angiogenic factor VEGF. Despite the crucial role of VEGF on residual tumor cells, foci progression and metastasis, the clinical impact of increased VEGF concentrations during the post-op period could not be evaluated from the current study. However, the immediate post-op period is not appropriate for any adjuvant chemotherapies and thus alternative approaches in order to control VEGF fluctuations may have a significant role on future progress of the disease. One such approach could be the use of the highest dose of Tinzaparin (or maybe of other LMWHs) for extended-duration, for example for up to post-op Day 30 or until the beginning of the administration of adjuvant agents. In our patients, only this strategy proved to be effective in restoring VEGF levels compared either to lower doses or to shorter periods of administration. Even though our results indicate a normalization of VEGF levels in group IV, chance findings could not be excluded given our small sample size. Further research, with larger sample sizes is needed to validate our current findings. Additionally, the relevance of the above thromboprophylaxis strategy to clinical outcomes, as well as, the hypothesis that mainly the surgical trauma-induced activation of platelets resulted in elevated post-op VEGF levels could be possibly answered in the context of a clinical trial with a more appropriate design.

\section{Abbreviations}

VEGF: Vascular Endothelial Growth Factor; CC: Colon Cancer; pre-op: preoperative; peri-op: perioperative; post-op: postoperative; LMWH: Low Molecular Weight Heparin; Day 0: preoperative day; Day 10: $10^{\text {th }}$ postoperative day; Day 30: $30^{\text {th }}$ postoperative day; CG: Consolidated Group; CG-CD: Consolidated Group-Conventional Dose; CG-HD: Consolidated Group-Highest Dose; CG-SD: Consolidated Group-Short Duration; CG-ED: Consolidated Group-Extended Duration; MC pts: Mucinous Patients; TF: Tissue Factor; TFPI: Tissue Factor Pathway Inhibitor.

\section{Acknowledgements}

The authors thank Dr Nikolaos Kolaitis, ex-Director of the Haematology Laboratory of the University Hospital of Ioannina, for his help with the laboratory analyses of the samples and his administrative support throughout the progression of the study.

\section{Competing Interests}

The authors have declared that no competing interest exists.

\section{References}

1. American Cancer Society USA. Key Statistics for Colorectal Cancer. https://www.cancer.org/cancer/colon-rectal-cancer/detection-diagnosis-sta ging/survival-rates/; Accessed 14.02.17

2. Wieder R: Insurgent micrometastases: sleeper cells and harboring the enemy. J Surg Oncol 2005; 89(4):207-210.

3. Li J, Jiang E, Wang X, Shangguan AJ, Zhang L, Yu Z: Dormant cells: the original cause of tumor recurrence and metastasis. Cell Biochem Biophys 2015; 72(2):317-320.

4. Udagawa T: Tumor dormancy of primary and secondary cancers. APMIS 2008; 116(7-8):615-28.

5. Holmgren L, O'Reilly MS, Folkman J: Dormancy of micrometastases: Balanced proliferation and apoptosis in the presence of angiogenesis suppression. Nature Medicine 1995; 1:149-153.

6. Naumov GN, Bender E, Zurakowski D, Kang SY, Sampson D, Flynn E, Watnick RS, Straume O, Akslen LA, Folkman J, Almog N: A model of human tumor dormancy: an angiogenic switch from the nonangiogenic phenotype. J Natl Cancer Inst 2006; 98(5):316-325.

7. Aguirre-Ghiso JA: Models, mechanisms and clinical evidence for cancer dormancy. Nature Reviews Cancer 2007; 7(11):834-846.

8. Shibuya M: Vascular endothelial growth factor (VEGF) and its receptor (VEGFR) signaling in angiogenesis: a crucial target for anti- and pro-angiogenic therapies. Genes \& Cancer 2011; 2(12):1097-1105.

9. Karayiannakis AJ, Syrigos $\mathrm{KN}$, Zbar A, Baibas $\mathrm{N}$, Polychronidis A, Simopoulos C, Karatzas G: Clinical significance of preoperative serum vascular endothelial growth factor levels in patients with colorectal cancer and the effect of tumor surgery. Surgery 2002; 131(5):548-555.

10. Werther K, Christensen IJ, Brunner N, Nielsen HJ and the Danish RANX05 colorectal cancer study group: soluble vascular endothelial growth factor 
levels in patients with primary colorectal carcinoma. Eur J Surg Oncol 2000; 26(7):657-662.

11. Kwon KA, Kim SH, Oh SY, Lee S, Han JY, Kim KH, Goh RY, Choi HJ, Park KJ, Roh MS, Kim HJ, Kwon HC, Lee JH: Clinical significance of preoperative serum vascular endothelial growth factor, interleukin-6, and C-reactive protein level in colorectal cancer. BMC Cancer 2010; 10:203-210.

12. De Vita F, Orditura M, Lieto E, Infusino S, Morgillo F, Martinelli E, Castellano P, Romano C, Ciardiello F, Catalano G, Pignatelli C, Galizia G: Elevated perioperative serum vascular endothelial growth factor levels in patients with colon carcinoma. Cancer 2004; 100(2):270-278.

13. Belizon A, Balik E, Horst P, Feingold D, Arnell T, Azarani T, Cekic V, Skitt R, Kumara S, Whelan RL: Persistent elevation of plasma vascular endothelial growth factor levels during the first month after minimally invasive colorectal resection. Surg Endosc 2008; 22(2):287-297.

14. De Gramont A, Cutsem EV, Schmoll HJ, Tabernero J, Clarke S, Moore MJ, Cunningham D, Cartwright TH, Hecht JR, Rivera F, Im SA, Bodoky G, Salazar R, Maindrault-Goebel F, Shacham-Shmueli E, Bajetta E, Makrutzki M, Shang A, André T, Hoff PM: Bevacizumab plus oxaliplatin-based chemotherapy as adjuvant treatment for colon cancer (AVANT): a phase 3 randomised controlled trial. The Lancet Oncology 2012; 13(12):1225-1233.

15. Norton L: Conceptual and practical implications of breast tissue geometry: toward a more effective, less toxic therapy. Oncologist 2005; 10(6):370-81.

16. Retsky M, Demicheli R, Hrushesky W, Baum M, Gukas I: Surgery triggers outgrowth of latent distant disease in breast cancer: An inconvenient truth? Cancers (Basel) 2010; 2(2):305-37.

17. Kumara HM, Feingold D, Kalady M, Dujovny N, Senagore A, Hyman N, Cekic V, Whelan RL: Colorectal resection is associated with persistent proangiogenic plasma protein changes. Postoperative plasma stimulates in vitro endothelial cell growth, migration, and invasion. Ann Surg 2009; 249(6):973-977.

18. Gould MK, Garcia DA, Wren SM, Karanicolas PJ, Arcelus JI, Heit JA, Samama CMl: Prevention of VTE in nonorthopedic surgical patients: antithrombotic therapy and prevention of thrombosis, 9th ed: American College of Chest Physicians Evidence-Based Clinical Practice Guidelines. Chest 2012; 141(2 Suppl):e227S-77S

19. Agnelli G, Caprini JA: The prophylaxis of venous thrombosis in patients with cancer undergoing major abdominal surgery: Emerging options. J Surg Oncol 2007; 96(3):265-272.

20. Borsig L: Heparin as an inhibitor of cancer progression. Progr Mol Biol Transl Sci 2010; 93:335-349.

21. Dipasco PJ, Misra S, Koniaris LG, Moffat FLJr. The thrombophilic state in cancer Part II: Cancer outcomes, occult malignancy, and cancer suppression. J Surg Oncol 2012; 106(4):517-523.

22. Amirkhosravi A, Mousa SA, Amaya M, Francis JL: Antimetastatic effect of tinzaparin, a low molecular-weight heparin. J Thromb Haemost 2003; 1(9):1972-1976.

23. Castoldi E, Brugge JM, Nicolaes GAF, Girelli D, Tans G, Rosing J. Impaired APC cofactor activity of factor $\mathrm{V}$ plays a major role in the APC resistance associated with the factor V Leiden (R506Q) and R2 (H1299R) mutations. BLOOD 2004; 103(11):4173-4179.

24. Marco A, Brocal C, Martirena F, Lucas J, Marco P: Clinical and biological factors that contribute to thrombin generation in prothrombin G20210A carriers: a case-control study in a single Thrombophilia Center. Thromb Res 2012; 129(5):e266-8.

25. Ahluwalia A, Jones MK, Matysiak-Budnik T, Tarnawski AS: VEGF and colon cancer growth beyond angiogenesis: does VEGF directly mediate colon cancer growth via a non-angiogenic mechanism? Curr Pharm Des 2014; 20(7):1041-1044

26. Takeda A, Shimada $H$, Imaseki $H$, Okazumi S, Natsume $T$, Suzuki $T$, Ochiai T: Clinical significance of serum endothelial growth factor in colorectal cancer patients: correlation with clinicopathological factors and tumor markers. Oncol Rep 2000; 7(2):333- 338.

27. Broll R, Erdmann H, Duchrow M, Oevermann E, Schwandner O, Markert U, Bruch HP, Windhövel U: Vascular endothelial growth factor (VEGF) - a valuable serum tumour marker in patients with colorectal cancer? Eur J Surg Oncol 2001; 27(1):37-44.

28. Banks RE, Forbes MA, Kinsey SE, Stanley A, Ingham E, Walters C, Selby PJ: Release of the angiogenic cytokine vascular endothelial growth factor (VEGF) from platelets: significance for VEGF measurements and cancer biology. Br J Cancer 1998; 77(6):956-964

29. Bambace NM, Holmes CE: The platelet contribution to cancer progression. J Thromb Haemost 2011; 9(2):237-249.

30. Lykke J, Nielsen HJ: Haemostatic alterations in colorectal cancer: Perspectives for future treatment. J Surgl Oncol 2004; 88(4):269-275.

31. Adam F, Bouton MC, Huisse MG, Jandrot-Perrus M: Thrombin interaction with platelet membrane glycoprotein Iba. Trends Mol Med 2003; 9(11):461.

32. Li N: Platelets in cancer metastasis: To help the "villain" to do evil. Int J Cancer 2016; 138(9):2078-2087.

33. Gerotziafas GT, Galea V, Mbemba E, Sassi M, Roman MP, Khaterchi A, van Dreden P, Japcowitz M, Lotz JP, Bernaudin JF, Fareed J, Hatmi M, Elalamy I: Effect of low molecular weight heparins and fondaparinux upon thrombin generation triggered by human pancreatic cancer cells BXPC3. Curr Vasc Pharmacol 2014; 12(6):893-902.
34. van den Berg YW, Osanto S, Reitsma PH, Versteeg HH: The relationship between tissue factor and cancer progression: insights from bench and bedside. Blood 2012; 119(4):924-932

35. Amirkhosravi A, Meyer T, Amaya M, Davila M, Mousa SA, Robson T, Francis JL: The role of tissue factor pathway inhibitor in tumor growth and metastasis. Semin Thromb Hemost 2007; 33(7):643-52.

36. Stevenson JL, Choi SH, Varki A: Differential metastasis inhibition by clinically relevant levels of heparins. Correlation with selectin inhibition, not antithrombotic activity. Clin Cancer Res 2005; 11(19):7003-7011.

37. Zhao W, McCallum SA, Xiao Z, Zhang F, Linhardt RJ: Binding affinities of vascular endothelial growth factor (VEGF) for heparin-derived oligosaccharides. Biosci Rep 2012; 32(1):71-81.

38. Consorti F, Lorenzotti A, Midiri G, Di Paola M: Prognostic significance of mucinous carcinoma of colon and rectum: A prospective case-control study. J Surg Oncol. 2000; 73(2):70-74. 